\title{
Development and in vitro evaluation of mucoadhesive patches of methotrexate for targeted delivery in oral cancer
}

\author{
BAO-ZHONG JIN $^{1}$, XIAO-QI DONG ${ }^{2}$, XIN XU $^{2}$ and FENG-HE ZHANG ${ }^{1}$ \\ ${ }^{1}$ Department of Oral Surgery, Stomatological Hospital of Shandong University, \\ Jinan, Shandong 250012; ${ }^{2}$ Department of Oral Surgery, The Second Affiliated Hospital of \\ Zhejiang University School of Medicine, Hangzhou, Zhejiang 310009, P.R. China
}

Received July 7, 2016; Accepted August 15, 2017

DOI: $10.3892 / \mathrm{ol} .2017 .7613$

\begin{abstract}
The present study focused on the development of a mucoadhesive patch of methotrexate (MTX) for targeted delivery in oral cancer. Initially, MTX-loaded liposomes were prepared using the thin film hydration method, and had a mean diameter of 105.7-137.4 $\mathrm{nm}$ and percentage entrapment efficiency of $54.6 \pm 3.5$. These liposomes were cast in optimized mucoadhesive film. The film was characterized by its release pattern, thickness, weight and percentage swelling index and the sustained release profile of the optimized film was evaluated. The developed liposomes and liposomes cast in the film formulation were evaluated for cytotoxicity in HSC-3 cells using an MTT assay, and a significant decrease in the half maximal inhibitory concentration of MTX was identified with the MTX-entrapped liposomal film, M-LP-F7. The results of the mitochondria-dependent intrinsic pathway demonstrated that there was significant mitochondrial membrane potential disruption with M-LP-F7 compared with the plain drug. M-LP-F7 increased the rate of apoptosis in HSC-3 cells by almost 3-fold. Elevated levels of reactive oxygen species provided evidence that M-LP-F7 exerts a pro-oxidant effect in HSC-3 cells.
\end{abstract}

\section{Introduction}

Oral cancer is one of the most common and invasive types of cancer, accounting for $\sim 5 \%$ of cancer mortalities worldwide. The management of oral cancer includes radiotherapy, chemotherapy and surgical excision, with which patients experience profound adverse effects. These undesired side effects of treatment occur due to the non-specific action of the therapeutic agents (1).

Correspondence to: Dr Feng-He Zhang, Department of Oral Surgery, Stomatological Hospital of Shandong University, 44 Wenhua West Road, Lixia, Jinan, Shandong 250012, P.R. China E-mail: drzhangfenghe@hotmail.com

Key words: mucoadhesive patch, liposomes, MTT assay, methotrexate, targeted delivery
Over the previous three decades, advances in drug delivery systems have reduced the undesired effects of chemotherapy. Furthermore, the progression of nanotechnology in cancer chemotherapy has promoted the innovation of diagnostic and treatment methods. Targeted therapy is the most desired treatment for oral cancer, aiming for specific site delivery and thereby lowering the side effects and levels of systemic toxicity. The delivery of therapeutics through nanodelivery systems consisting of polymers or lipids have demonstrated increased solubility, stability and bioavailability, accumulating even inside tumor cells (2).

Novel drug delivery systems tackle the issues associated with local oral drug delivery and overcome various challenges, including lower drug absorption from the oral mucosa and prompt clearance of the drug from the site of absorption with saliva and induced mechanical stress, and poor patient compliance due to unpleasant taste (2). In addition, the most prominent disadvantage of conventional chemotherapy used to treat oral cancer is its high systemic toxicity and poor target specificity at cancer sites (2). Liposomes exhibit marked potential as a carrier system for therapeutically active agents. Due to their attributes, including biodegradability, biocompatibility, low toxicity and the ability to encapsulate hydrophilic and hydrophobic drugs, liposomes have gained attention in studies investigating targeted delivery systems for cancer agents. They function by directly targeting the tumor sites (3), and have been developed as a multifunctional drug carrier as they are able to prolong systemic drug circulation, enhance drug accumulation at the target tissue, increase levels of cellular internalization and provide organelle-specific drug delivery (4).

These strategies have developed from controlled drug delivery systems to local controlled drug delivery systems for oral cancer therapy. Previous developments in drug delivery through the oral mucosa via bio-adhesive polymers gained notable attention. The oral cavity offers a unique route for drug delivery, due to its relatively increased permeability to drugs, drug absorption, avoidance of hepatic first pass metabolism and enhances bioavailability (5). The preferred forms of administration via the oral cavity are adhesive gels, films, patches and tablets intended for local drug delivery (6-9).

Oral strips were developed for local application via the tongue or buccal cavity. Mucoadhesive buccal patches offered benefits compared with conventional methods (10). Due to the 
short residence time of oral gels in oral cavity, buccal patches have advantages of increased residence time and drug release (5). For the development of controlled-release mucoadhesive buccal patches, the time of contact of drug and tumor cells present in oral mucosa are important aspects for consideration. Though oral mucosa is more permeable compared with skin, the buccal mucosa exhibits a limited permeation to drugs. The buccal mucosa exhibits dual transport mechanisms, including a paracellular transport mode for hydrophilic compounds and large molecules, and a transcellular transport mode for lipophilic drugs that typically pass through the lipid bilayer (11). To overcome the permeation barrier in oral mucosa, the residence time of mucoadhesive buccal patches on the buccal mucosa has been increased to enhance the drug partitioning to the target tissue. Thereby, such methods may effectively contribute to the development of a sustained and targeted delivery system for drugs designed for tissues in the oral cavity $(5,12,13)$.

Previous studies on mucoadhesive polymers demonstrated that buccal tablets prepared using chitosan exhibited excellent mucoadhesive properties and a high capacity for drug permeation through the buccal mucosa $(11,14,15)$. Mishra et al (16) developed buccal patches, which consisted of a polymer combination of hydroxypropyl methylcellulose (HPMC), poly (vinyl alcohol) (PVA) and sodium carboxymethyl cellulose. These patches were evaluated to determine their physicochemical properties and in vitro release profile. The results highlighted the bio-adhesive performance of PVA and HPMC. Additionally, HPMC and PVA exhibited an extended release of almost $40 \%$ of the drug in $12 \mathrm{~h}$. Abbasi et al (17) formulated a doxorubicin-methotrexate (MTX)-loaded nanoparticle to attenuate oral cancer growth, and evaluated them in an oral squamous cell carcinoma (OSCC) rat model. Additionally, this study group identified that the downregulation of matrix metalloproteinase 2 and receptor tyrosine-protein kinase ErbB-2 gene expression in an OSCC rat model were responsible for the clinical outcomes observed $(17,18)$. MTX is a commonly used anticancer agent for various types of cancer, including colon, breast, skin and head and neck cancer (19-21). Due to the high toxicity of MTX, various strategies have been attempted to formulate an effective delivery of MTX with reduced side effects. Dhanikula et al (22) developed modified polyester-co-polyether dendrimers that encapsulated MTX and performed a release study. Furthermore, another study investigated the controlled release of MTX from intercalated nanocomposites (23). In the present study, a controlled-release buccal delivery system for MTX was designed, where MTX was loaded into a liposome system, increasing the retention time and release of MTX within the oral cavity, thereby prolonging the therapeutic effect. The lipid vesicles enhance anticancer efficiency of MTX and composite chitosan-HPMC-PVA as a buccal patch, through efficient delivery of MTX at the oral mucosal membrane. Consequently, a targeted delivery system for MTX was designed, resulting in site-specific treatment of oral cancer. The cytotoxicity results of the present study on HSC-3 cells suggest that apoptosis is the underlying mechanism of action. Mitochondrial depolarization and pro-oxidant effects were the primary events observed in the MTX-chitosan-HPMC-PVA composite liposomes that induced cell apoptosis in HSC-3 cells.

\section{Materials and methods}

Materials. Phosphatidylcholine (PC) from soybean lecithin was purchased from Sigma-Aldrich; Merck KGaA (Darmstadt, Germany). MTX was procured from Wako Pure Chemical Industries, Co., Ltd. (Osaka, Japan). Cholesterol (CL), HPMC, hydroxyethyl cellulose (HEC), PVA, poly (ethylene glycol) (PEG) and chitosan $(\mathrm{CH})$ were all purchased from Sigma-Aldrich; Merck KGaA.

Cell culture. The human oral squamous cell carcinoma HSC-3 cell line was purchased from American Type Culture Collection (Manassas, VA, USA). Cells were cultured in Dulbecco's Modified Eagle's Medium (DMEM; Sangon Biotech Co., Ltd., Shanghai, China) containing 10\% fetal bovine serum (Sangon Biotech Co., Ltd.), $1 \%$ penicillin streptomycin $(10,000 \mathrm{U} / \mathrm{ml}$ penicillin and $10 \mathrm{mg} / \mathrm{ml}$ streptomycin; Sangon Biotech Co., Ltd.) and $1 \%$ glutamine in tissue culture flasks, at $37^{\circ} \mathrm{C}, 5 \%$ $\mathrm{CO}_{2}$ and $95 \%$ humidity.

Preparation of MTX-loaded liposomes. MTX-loaded liposomes were formulated using the thin film hydration method using various molar ratios of PC in the presence of CL. Different molar ratios of PC/CL were mixed with PEG 400 and dissolved in 95\% diethyl ether solvent and 99\% chloroform (Sigma-Aldrich; Merck KGaA; 1:1). The solution was transferred to a round-bottomed flask and the thin lipid layer was obtained by evaporating the solution in a rotary evaporator $(50 \mathrm{rev} / \mathrm{min})$ at $40^{\circ} \mathrm{C}$. The lipid film was then lyophilized overnight at $25^{\circ} \mathrm{C}$ to remove the remaining organic solvent. This lipid film was rehydrated with acetate buffer solution ( $\mathrm{pH} 5.5)$ containing MTX solutions of various concentrations (0.5 and $1.0 \%$, w/w), and the resulting solution was agitated at $250 \mathrm{rev} / \mathrm{min}$ for $1 \mathrm{~h}$ at $25^{\circ} \mathrm{C}$ to obtain stable and homogeneous liposomes. Three MTX-entrapped liposomes (as denoted by 'M-LP', 'M-LF' and 'M-LN') were prepared, the composition of which described in Table I. These MTX-entrapped liposomes (M-LP, M-LF, M-LN) exhibited different concentrations of PC and CL (Table I) and were sonicated in an ice bath (30 min intervals) and extruded through membrane filters in five cycles (twice through a nylon filter at $0.45 \mathrm{~mm}$ and three times through a cellulose acetate filter at $0.20 \mathrm{~mm}$ ) to reduce particle size in order to be suitable for mucoadhesive delivery systems (24). The dispersions of M-LF, M-LN and M-LP were maintained at $4^{\circ} \mathrm{C}(25)$ and were characterized to obtain the final optimized MTX-loaded liposomes.

Colloidal characterization of liposomes. The particle size, polydispersity index and zeta potential of M-LF, M-LN and M-LP were estimated using a Malvern Zetasizer Nano ZS ${ }^{\mathrm{TM}}$ (Malvern Instruments, Malvern, UK). The liposomes were diluted 20 times with deionized water and evaluated. Morphological surface characteristics of the LPs were examined using transmission electron microscopy (Jeol-100CX; JEOL, Ltd., Tokyo, Japan) operating at $100 \mathrm{kV}$. A drop of sample (5-10 $\mu \mathrm{l})$ was positioned on a 300-mesh carbon-coated copper grid to form a thin liquid film. Excess sample was removed by gently touching the grid with a filter paper. The negative staining of the films was performed using $1 \%$ (w/v) uranyl acetate at $25^{\circ} \mathrm{C}$ for $60 \mathrm{sec}$ by placing a drop of stain to the grid. After $10 \mathrm{sec}$ the excess stain was removed by 
Table I. Characterization of MTX-loaded liposomes.

\begin{tabular}{|c|c|c|c|c|c|c|}
\hline Formulation & $\begin{array}{l}\text { PC:CL: } \\
\text { PEG } 400\end{array}$ & MTX: lipid & $\begin{array}{l}\text { Particle } \\
\text { size, } \mathrm{nm}\end{array}$ & PDI & $\begin{array}{c}\text { Zeta } \\
\text { potential, } \mathrm{mV}\end{array}$ & $\begin{array}{l}\text { Entrapment } \\
\text { efficiency, \% }\end{array}$ \\
\hline M-LF & $55: 40: 5$ & $1: 20$ & $105.7 \pm 5.5$ & 0.13 & $8.1 \pm 3.7$ & $54.6 \pm 3.5$ \\
\hline M-LN & 60:35:5 & $1: 20$ & $111.8 \pm 2.8$ & 0.34 & $22.4 \pm 1.2$ & $67.2 \pm 1.5$ \\
\hline M-LP & $60: 35: 5$ & $1: 10$ & $137.4 \pm 2.6$ & 0.31 & $36.0 \pm 3.1$ & $73.4 \pm 1.7$ \\
\hline
\end{tabular}

Data are presented as the mean \pm standard deviation $(n=3) . F, N$ and $\mathrm{P}$ denote the identity of the MTX-entrapped liposomes with distinct compositions. MTX, methotrexate; PC, phosphatidylcholine; CL, cholesterol; PEG, poly(ethylene glycol); PDI, polydispersity index.

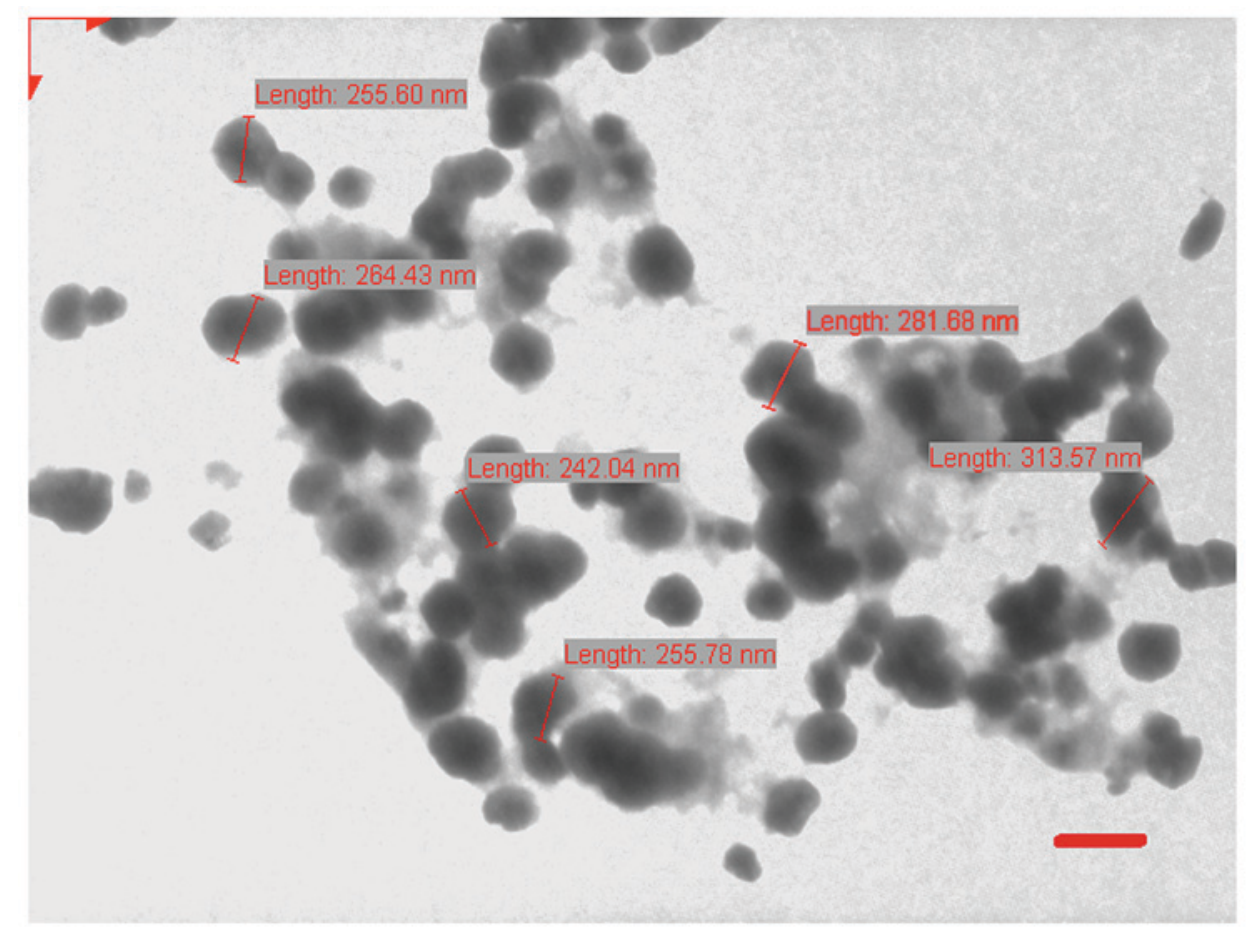

Figure 1. Morphological characteristics of liposomes (M-LP) examined using transmission electron microscopy. The micrographs demonstrated that the majority of liposomes have a diameter between 200 and $300 \mathrm{~nm}$. Scale bar represents $200 \mathrm{~nm}$.

touching the edge to a filter paper. The extra staining solution was cleared with a filter paper and allowed to air-dry. The stained films were photographed using transmission electron microscope. All procedures were carried out at $25^{\circ} \mathrm{C}$ (Fig. 1).

High-performance liquid chromatography (HPLC). MTX-loaded liposomes were quantified using HPLC. The samples were analyzed on a HPLC system (Waters Alliance, Milford, MA, USA) using an RP C18 column (250x4.6 mm, particle size $5 \mu \mathrm{m}$; Phenomenex, Torrance, CA, USA) at $25^{\circ} \mathrm{C}$. The mobile phase used for MTX detection and quantification was acetate buffer [acetonitrile solution (90:10) at flow rate of $1 \mathrm{ml} / \mathrm{min}$ and $20 \mu \mathrm{l}$ injection volume], and the wavelength for detection was $302 \mathrm{~nm}$ using Waters Tunable UV/Visible Absorbance Detector (Waters Alliance) (26).

Determination of drug entrapment efficiency. MTX-loaded liposomes were evaluated for percentage entrapment efficiency (\%EE) by vesicle lysis with $0.2 \%$ (v/v) Triton $^{\mathrm{TM}} \mathrm{X} 100$ solution, and the lysed suspension was centrifuged at 12,500 $\mathrm{x} \mathrm{g}$ and $5^{\circ} \mathrm{C}$ for $15 \mathrm{~min}$. The supernatant was collected for analysis of the MTX content via the aforementioned HPLC method. The experiment was performed in triplicate. Experimental and theoretical percentages of MTX loading were calculated using the following formula: Percentage entrapment=[amount of drug in liposomes/total amount of drug added]x100. Based on the highest encapsulation efficiency with lowest lipid concentration, M-LP was selected to be loaded into the mucoadhesive films.

Preparation of buccal mucoadhesive films. A solvent casting method using various polymer solutions formulated the buccal mucoadhesive films: CH (1\% w/v); HPMC (1\% w/v); PVA $(1 \% \mathrm{w} / \mathrm{v})$. The casting solution was a mixture of three ratios of these polymers left overnight at room temperature to ensure a clear, bubble-free viscous mixture. The mixture was cast into a glass Petri dish and allowed to dry at room temperature $\left(25^{\circ} \mathrm{C}\right)$ and $40-45 \%$ humidity for $24-48 \mathrm{~h}$ until a flexible film was formed. The films with different compositions were cast as F1 to F7 and their characteristics are presented in Table II. The films were optimized for physical characteristics including 
Table II. Composition of mucoadhesive buccal films.

\begin{tabular}{|c|c|c|c|c|}
\hline Formulation & Chitosan, \% w/w & HPMC, \% w/w & $\mathrm{PVA}, \% \mathrm{w} / \mathrm{w}$ & Physical characteristics of film \\
\hline F1 & 99 & 0 & 0 & Stiff and opaque, malleable \\
\hline $\mathrm{F} 2$ & 75 & 24 & 0 & Stiff and opaque, malleable \\
\hline F3 & 25 & 74 & 0 & Less stiff and opaque, malleable \\
\hline $\mathrm{F} 4$ & 50 & 49 & 0 & Little flexibility but opaque, malleable \\
\hline F5 & 75 & 0 & 24 & Low flexibility translucent, malleable \\
\hline F6 & 50 & 0 & 49 & Low flexibility, translucent, malleable \\
\hline F7 & 25 & 50 & 24 & Soft, flexile, translucent and malleable \\
\hline
\end{tabular}

HPMC, hydroxypropyl methylcellulose; PVA, poly(vinyl alcohol).

thickness, average weight and percentage swelling (Table III). Other parameters including softness, flexibility, translucence and malleability were also taken into account. The F7 film was identified to be the most translucent and flexible, with highest swelling index and average thickness. Thus, the F7 film was selected to prepare the MTX- and M-LP-loaded mucoadhesive film. The MTX and M-LP-dispersion was added to the polymer dispersion under continuous stirring to form M-F7 and M-LP-F7 polymeric suspensions, respectively. The PEG 400 served as a plasticizer for the films. The gels formed were maintained in desiccators overnight at room temperature to ensure a bubble-free and clear casting solution, and then cast into glass Petri dishes. The cast polymer solution was allowed to dry at room temperature. Dried M-F7 and M-LP-F7 films measuring $1 \mathrm{~cm}^{2}$ were packed in aluminum foil, and stored in desiccators at room temperature (27).

\section{Pharmaceutical evaluation of mucoadhesive films}

Weight uniformity. Weight uniformity was determined by weighing six films of each set individually, and the average weight was determined with the standard deviation.

Film thickness. The thickness of the circular films was measured at five distinct positions (center and four places at the circumference) at the surface of each film using a screw gauge, and the mean value was used as the film thickness.

Determination of the swelling indices of films in distilled water. The swelling indices of the films were determined: Films were coated with ethyl cellulose on the lower base so that sticking of film to dish was avoided. The films were re-weighed (W1) and allowed to swell in Petri dishes containing $10 \mathrm{ml}$ of distilled water. The films were incubated at $30^{\circ} \mathrm{C}$ in distilled water for $30 \mathrm{~min}$ and stored at room temperature. Following this, the films were re-weighed (W2), and the percentage of swelling was calculated using the following formula: Swelling index $=(\mathrm{W} 2-\mathrm{W} 1 / \mathrm{W} 1) \times 100(28)$.

In vitro release of compound from different film. In vitro release of MTX from M-LP, M-F7 and M-LP-F7 were assessed using the dialysis bag method at $37 \pm 0.5^{\circ} \mathrm{C}$. The formulations M-LP, M-F7 and M-LP-F7 were placed in the dialysis bag separately and suspended in $20 \mathrm{ml}$ simulated saliva ( $\mathrm{pH} 6.75 \pm 0.05)$ in separate beakers (simulated saliva was prepared by dissolving
Table III. Physical characteristics of mucoadhesive films at room temperature.

\begin{tabular}{lccc}
\hline Formulation & $\begin{array}{c}\text { Thickness } \pm \\
\text { SD, mm }\end{array}$ & $\begin{array}{c}\text { Average } \\
\text { weight } \pm \text { SD, g }\end{array}$ & Swelling, \% \\
\hline F1 & $0.45 \pm 0.023$ & $1.063 \pm 0.076$ & 93.2 \\
F2 & $0.44 \pm 0.022$ & $0.953 \pm 0.126$ & 85.4 \\
F3 & $0.36 \pm 0.031$ & $0.532 \pm 0.018$ & 62.13 \\
F4 & $0.46 \pm 0.01$ & $0.678 \pm 0.07$ & 74.3 \\
F5 & $0.52 \pm 0.025$ & $0.872 \pm 0.03$ & 76.5 \\
F6 & $0.55 \pm 0.015$ & $0.77 \pm 0.015$ & 94.6 \\
F7 & $0.42 \pm 0.037$ & $0.681 \pm 0.024$ & 96.5 \\
\hline
\end{tabular}

SD, standard deviation.

$2.38 \mathrm{~g} \mathrm{Na}_{2} \mathrm{HPO}_{4}, 0.19 \mathrm{~g} \mathrm{KH}_{2} \mathrm{PO}_{4}$ and $8.0 \mathrm{~g} \mathrm{NaCl}$ in $1,000 \mathrm{ml}$ distilled water). Each beaker was maintained on a magnetic stirrer rotating at $250 \mathrm{rpm}$. After $0.5,1,2,3,4,5$ and $6 \mathrm{~h}$ intervals, a $0.5 \mathrm{ml}$ sample was taken from each beaker, filtered through a Millipore filter $(0.45 \mathrm{~mm})$ and analyzed using HPLC. A similar volume of fresh medium was added to each sample immediately following this to maintain a constant medium volume.

In vitro cell growth-inhibition assay. The cytotoxicity of MTX, M-LP and M-LP-F7 was evaluated in HSC-3 cells by an MTT assay. The HSC-3 cells were cultured in 96-well plates in DMEM media at a density of $29 \times 10^{3}$ cells/well and were incubated for $24 \mathrm{~h}$ at $37^{\circ} \mathrm{C}$. Following this, cells were treated with MTX, M-LP and M-LP-F7 at equivalent concentrations of $\operatorname{MTX}(50,100$ and $200 \mu \mathrm{g} / \mathrm{ml})$ and incubated at $37^{\circ} \mathrm{C}$ for an additional $24 \mathrm{~h}$. At the end of the incubation time, the cells were incubated again at $37^{\circ} \mathrm{C}$ with MTT reagent $(8 \mu 1$, $5 \mathrm{mg} / \mathrm{ml}$ ) for $2 \mathrm{~h}$, and finally optical density was read with an ELISA plate reader (Molecular Devices, LLC, Sunnyvale, CA, USA) at a wavelength of $540 \mathrm{~nm}$. The half maximal inhibitory concentration $\left(\mathrm{IC}_{50}\right)$ values were calculated from the mean absorbance at $490 \mathrm{~nm}$ (29).

\section{Cell apoptosis study}

Flow cytometric analysis of apoptosis in HSC-3 cells using Annexin V-fluorescein isothiocyanate (FITC)/propidium 
iodide (PI) assay. The apoptotic effects of MTX and M-LP-F7 was studied in HSC-3 cells by quantitative discrimination of live cells, early apoptotic, late apoptotic and necrotic cells using dual staining with the Annexin V-FITC/PI dye. Briefly, the HSC-3 cells were treated with MTX and M-LP-F7 having an equivalent concentration of $70 \mu \mathrm{g} / \mathrm{ml}$ MTX and incubated at $37^{\circ} \mathrm{C}$ for $24 \mathrm{~h}$. Following incubation, cells were washed and collected in PBS and stained with Annexin V-FITC/PI according to the manufacturer's protocol (kit supplied by Sangon Biotech Co., Ltd.) cells were trypsinized (1x trypsin) and collected via centrifugation at $300 \mathrm{x} \mathrm{g}$ at $25^{\circ} \mathrm{C}$ for $5 \mathrm{~min}$. Cells were suspended in $400 \mu 1 \mathrm{1x}$ Annexin $\mathrm{V}$ solvent. In total, $5 \mu \mathrm{l}$ Annexin V-FITC was added to the suspension and incubated for $15 \mathrm{~min}$ at $2-8^{\circ} \mathrm{C}$. Furthermore, $10 \mu 1 \mathrm{PI}$ was added to the solution and incubated for $5 \mathrm{~min}$ at $2-8^{\circ} \mathrm{C}$. Flow cytometric analysis was carried out immediately following the addition of PI using a FACS Calibur instrument (BD Biosciences, San Jose, CA, USA). The data were analyzed using FlowJo software (version 7.6; BD Biosciences).

Determination of mitochondrial membrane potential ( $\Delta \Psi \mathrm{m})$ in HSC-3 cells using flow cytometry. The apoptotic mechanism was investigated in terms of change in $\Delta \Psi \mathrm{m}$. The JC1 dye was utilized to monitor changes in $\Delta \Psi \mathrm{m}$; in live cells, JC1 fluoresces red, whereas in dead cells the florescence changes to green. The cells were cultured in the same conditions as in the cell apoptosis study. Following $24 \mathrm{~h}$ of treatment with equivalent MTX concentration of $70 \mu \mathrm{g} / \mathrm{ml}$ for MTX and M-LP-F7, the HSC-3 cells were collected and incubated at $37^{\circ} \mathrm{C}$ with $1 \mu \mathrm{JC} 1$ dye in $500 \mu \mathrm{l}$ incubation buffer for $1 \mathrm{~h}$, followed by centrifugation at $300 \mathrm{x} g$ for $10 \mathrm{~min}$ at room temperature. The cell pellet was washed with PBS and reconstituted with $500 \mu \mathrm{l}$ incubation buffer and analyzed using flow cytometry $(30,31)$.

Detection of intracellular reactive oxygen species (ROS) in HSC-3 cells using flow cytometry by 2',7'-dichlorofluorescin diacetate (DCFDA) assay. The levels of intracellular ROS were evaluated to determine the effect of M-LP-F7 on ROS-mediated apoptosis in HSC-3 cells. The HSC-3 cells were treated with M-LP-F7 at an equivalent concentration of $\operatorname{MTX}(70 \mu \mathrm{g} / \mathrm{ml})$ for $24 \mathrm{~h}$ in order to the determine extent of ROS production. Cells were extracted in PBS, washed three times and incubated at $37^{\circ} \mathrm{C}$ with $5 \mu \mathrm{M}$ DCFDA for $30 \mathrm{~min}$. Subsequently, cells were centrifuged at $300 \mathrm{x}$ g for $10 \mathrm{~min}$ and re-suspended in $500 \mu \mathrm{l}$ PBS prior to flow cytometry analysis. Hydrogen peroxide was used as the positive control $(30,31)$.

Statistical analysis. All results are expressed as the mean \pm standard deviation $(n=3)$. Differences between formulations were compared with one-way analysis of variance followed by the Tukey-Kramer multiple comparisons test, using Graph Pad Prism (version 5; GraphPad Software, Inc., La Jolla, CA, USA). P $<0.05$ was considered to indicate a statistically significant difference.

\section{Results}

Size, polydispersity index, and zeta potential of MTX-loaded liposomes $(M-L F, M-L N, M-L P)$. The average sizes of MTX-loaded liposomes (M-LF, M-LN, M-LP) varied with the variation in the ratio of lipids. The mean size of liposomes increased with the ratio of PC. Additionally, the percentage of MTX used for loading also affected the liposome size. The size of liposomal formulations ranged between 105.7 and $137.4 \mathrm{~nm}$ (Table I). For M-LF liposomes with MTX/lipid ratio of 1:20, the vesicle size was $105.7 \pm 5.5 \mathrm{~nm}$, zeta potential was $8.1 \pm 3.7 \mathrm{mV}$ with $\% \mathrm{EE} 54.6 \pm 3.5$. Increases in the ratio of $\mathrm{PC}$ in M-LN and M-LP, resulted in increases in the size of vesicles to between $111.8 \pm 2.8$ and $137.4 \pm 2.6 \mathrm{~nm}$, respectively. The zeta potential of liposomes also changed towards more positive values, and \%EE was augmented in M-LN and M-LP.

Characteristics of LP-film. The three polymers, CH, HPMC and PVA, were used for the development of mucoadhesive buccal films. The casting solution was a blend of these in various ratios (Table II). The physical attributes of the films were characterized and the results are summarized in Table III. As the chitosan polymeric solution was viscous, the developed films were pale yellow colored, opaque and slightly hard, but varying the ratio of $\mathrm{CH}$ along with HPMC and PVA improved the texture of the film and malleability. The HEC film formulation was difficult to remove from the casting surface and possessed a non-homogeneous surface due to the presence of entrapped air bubbles. The polymeric solutions of films containing PVA were less viscous and air bubbles were easily removed, creating a homogeneous film surface. These films were transparent, malleable and flexible, but were not soft and sharp edges were formed on folding. The concentration of PVA was optimized in $\mathrm{CH}$ and HPMC polymer combinations in order to obtain mucoadhesive films which possessed the desired softness and flexibility.

The thickness and weight of the formulations varied from $0.36-0.55 \mathrm{~mm}$ and $0.532-1.063 \mathrm{~g}$, respectively. The composition of the casting solution decided the thickness and weight of the film formulations. As demonstrated in Table III, the film formulations with a high concentration of $\mathrm{CH}$ exhibited increased weight and thickness compared with the other two polymers. The thickness of the films with different concentrations of polymers designated (F1 to F7) were arranged into the following order: $\mathrm{F} 1>\mathrm{F} 5>\mathrm{F} 2>\mathrm{F} 6>\mathrm{F} 4>\mathrm{F} 7>\mathrm{F} 3$. The weights of the films were arranged into the following order: $\mathrm{F} 1>\mathrm{F} 5>\mathrm{F} 2>\mathrm{F} 6>\mathrm{F} 7>\mathrm{F} 4>\mathrm{F} 3$.

The percentage swelling index refers to the volume subsequent to swelling in aqueous liquid under pre-determined conditions including the weight of the film, temperature and humidity of the environment. The percentage swelling index of the films was evaluated by comparing the pre-weight and post weight of each film, and the effect of swelling on particle release. The mucoadhesive polymers swelled when exposed to water, and a consequently a weak network was formed at the bio-adhesive sites, resulting in bio-adhesion. The percentage swelling of the mucoadhesive film containing different polymers is summarized in Table III. The order of the percentage swelling of films with different concentration of polymers designated from F1 to F7 was: F7>F6 $>$ F1 $>$ F2 $>$ F5 $>$ F4 $>$ F3.

The in vitro release profiles of M-F7,M-LP and M-LP-F7 were compared in Fig. 2. As indicated, M-LP-F7 demonstrated a slower release profile compared with the M-F7 and M-LP films. The sustained release profile of M-LP-F7 may be attributed to the fact that $\mathrm{CH}$ maintained the integrity of the incorporated 


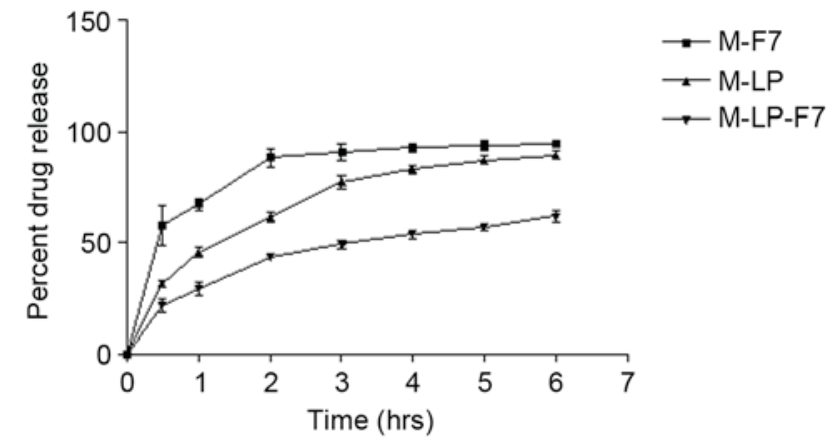

Figure 2. Release of MTX from M-F7, M-LP and M-LP-F7. M-LP-F7 demonstrated a slower release profile compared with the M-F7 and M-LP films. MTX, methotrexate; M-LP, MTX-entrapped liposomes; M-F7, MTX laden buccal mucoadhesive film; M-LP-F7, MTX-entrapped liposomes laden buccal mucoadhesive film 7 .

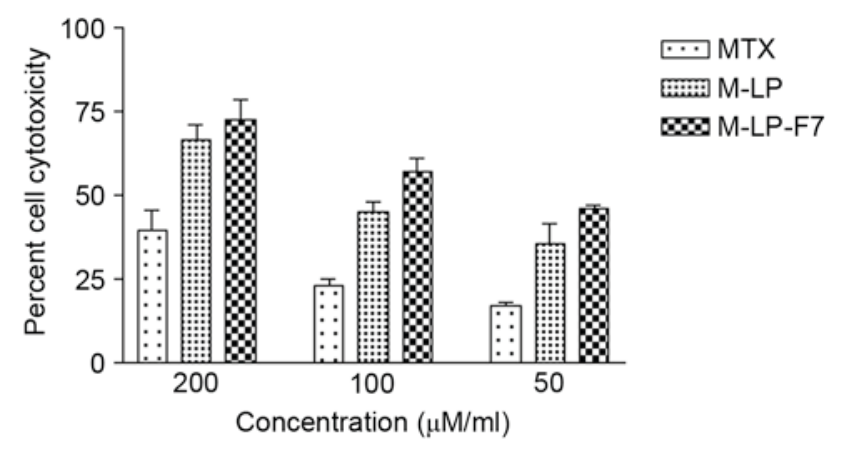

Figure 3. Cell cytotoxicity was assessed using an MTT assay in HSC-3 cells. The MTX, M-LP revealed decreased cytotoxicity compared with M-LP-F7 at all concentrations when incubated with HSC-3 cells for $24 \mathrm{~h}$. M-LP-F7 revealed significantly $(\mathrm{P}<0.05)$ enhanced toxicity vs. M-LP and MTX. MTX, methotrexate; M-LP, MTX-entrapped liposomes; M-F7, MTX laden buccal mucoadhesive film; M-LP-F7, MTX-entrapped liposomes laden buccal mucoadhesive film 7 .

vesicles. After 6 h, M-LP-F7 released $~ 52 \%$ of drug, whereas M-F7 and M-LP released 73 and 81\%, respectively.

\section{Cell culture assays}

In vitro cytotoxicity study of MTX-loaded liposome-based films. The developed liposomes and liposomes cast in film formulation were evaluated for cytotoxicity on HSC-3 cells by MTT assay. MTX, M-LP and M-LP-F7 were incubated with HSC-3 cells at equivalent concentration of MTX ranging from 50 to $200 \mu \mathrm{g} / \mathrm{ml}$ for $24 \mathrm{~h}$. The $\mathrm{IC}_{50}$ values of MTX, M-LP and M-LP-F7 were compared, and a significant decrease in the $\mathrm{IC}_{50}$ of MTX was identified with $75 \mu \mathrm{g} / \mathrm{ml}$ M-LP-F7, as compared with $142 \mu \mathrm{g} / \mathrm{ml}$ M-LP and $180 \mu \mathrm{g} / \mathrm{ml}$ MTX alone (Fig. 3).

Apoptosis study in HSC-3 cells. The apoptotic effect of MTX, alone and in formulation, on HSC-3 cancer cells was determined via flow cytometry in order to detect the total live cell and apoptotic cell populations. Apoptosis was evaluated using a Annexin V-FITC/PI assay and dual staining with Annexin V and PI. Fig. 4A demonstrates that the upper right quadrant represents Annexin V-positive and PI-positive cells. The M-LP-F7 system increased the rate of HSC-3 cell apoptosis almost 3-fold (Fig. 4A).
Assay of intracellular ROS. Intracellular ROS are responsible for apoptosis in cancer cells; therefore, raising ROS levels in cancer cells is an approach for cancer therapy, termed the pro-oxidant effect. The pro-oxidant effect of M-LP-F7 was measured using a DCFDA assay. The results demonstrated marked ROS generation by M-LP-F7 compared with $\mathrm{H}_{2} \mathrm{O}_{2}$, with an almost $68 \%$ shift in the M2 peak, as demonstrated in Fig. 4B. Increased ROS levels provided evidence that M-LP-F7 exerts a pro-oxidant effect in HSC-3 cells.

Mitochondria-dependent intrinsic pathway as evaluated using a JCl assay. Mitochondrial depolarization is considered the intrinsic pathway for apoptosis; therefore, changes in $\Delta \Psi \mathrm{m}$ were evaluated using the florescent probe JC1. The effect of M-LP-F7 in HSC-3 cells was evaluated in terms of $\Delta \Psi \mathrm{m}$ disruption. The results indicated that the $\Delta \Psi \mathrm{m}$ disruption by M-LP-F7 was significant $(\mathrm{P}<0.05)$, and increased 2-fold in comparison with MTX alone following treatment with an equivalent concentration of MTX (70 $\mu \mathrm{g} / \mathrm{ml}$; Fig. 4C).

\section{Discussion}

MTX is a common drug with antifolate characteristics that is used for cancer chemotherapy. In addition to the therapeutic effects, it also causes unavoidable adverse effects. Various strategies have been suggested to reduce the toxic effects of MTX. Amongst these, the design of lipid-based and polymer-based carriers has been successful. The present study aimed to develop a lipid-based local delivery system for oral cancer. To achieve this, MTX-loaded lipid vesicles were designed and cast in a polymer solution to develop liposome-laden mucoadhesive films. This formulation system has various benefits, as the liposomes contribute to enhancing MTX bioavailability, as well as the site-specific delivery offered by mucoadhesive patches. The lipid vesicles were constructed in a size range of between $105.7 \pm 5.5$ and $137.4 \pm 2.6 \mathrm{~nm}$. The size of the vesicles was increased with the increasing concentration of PC. The zeta potential also varied with the change in the $\mathrm{PC}$ and $\mathrm{CL}$ concentration, and size uniformity was attained at a drug/polymer ratio of 1:10 in the formulation M-LP. The increased \%EE in M-LP and M-LN depended on various factors, including the method of preparation, vesicle size and lamellarity, drug physicochemical properties, lipid concentration and drug-lipid interactions (32-34). M-LP was identified as the optimized MTX-loaded liposome based on increased \%EE with lowest drug/polymer ratio. M-LP was used for the preparation of liposome-laden buccal mucoadhesive films.

Following the successful fabrication of MTX-loaded liposomes (M-LP), a polymeric system was utilized for formulating MTX liposome-loaded buccal films. The hydrophilic polymers HEC, HPMC and hydrogel $\mathrm{CH}$ were used in different combinations and evaluated to improve the film formulation properties. A CH/HPMC/PVA polymer composition of 25:50:24 was optimized to develop soft, flexible and malleable films.

Mucoadhesive polymers, including HPMC, HEC and $\mathrm{CH}$, have potential in the development of film formulation as they form a swellable polymeric matrix to control drug release. 
A
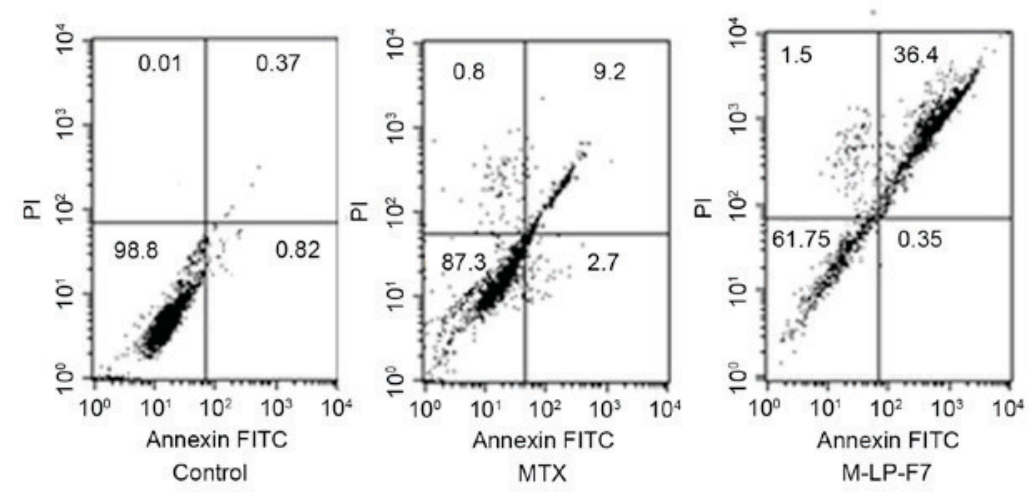

$B$
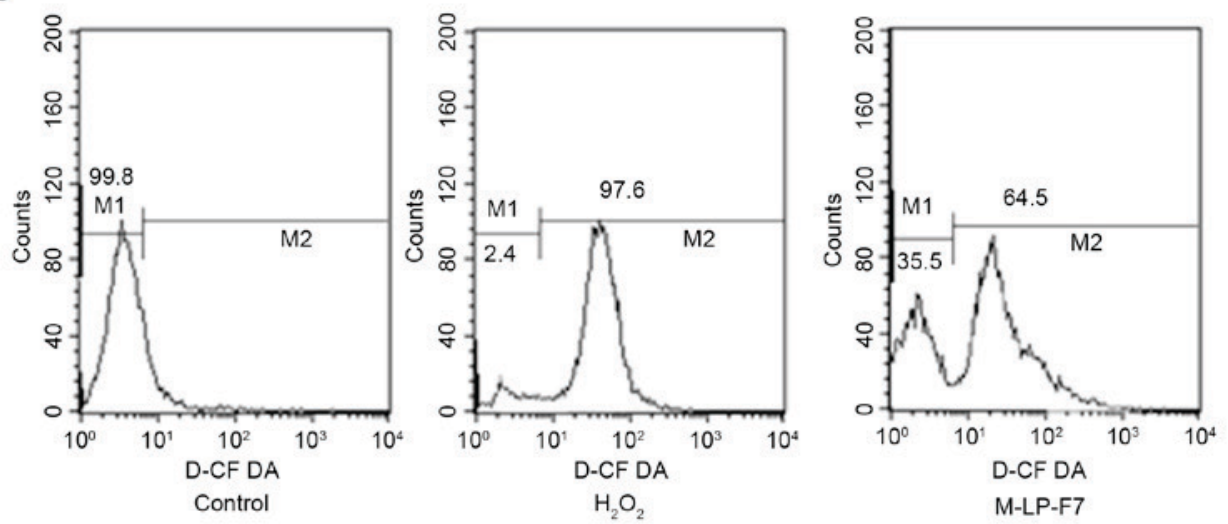

$\mathrm{C}$
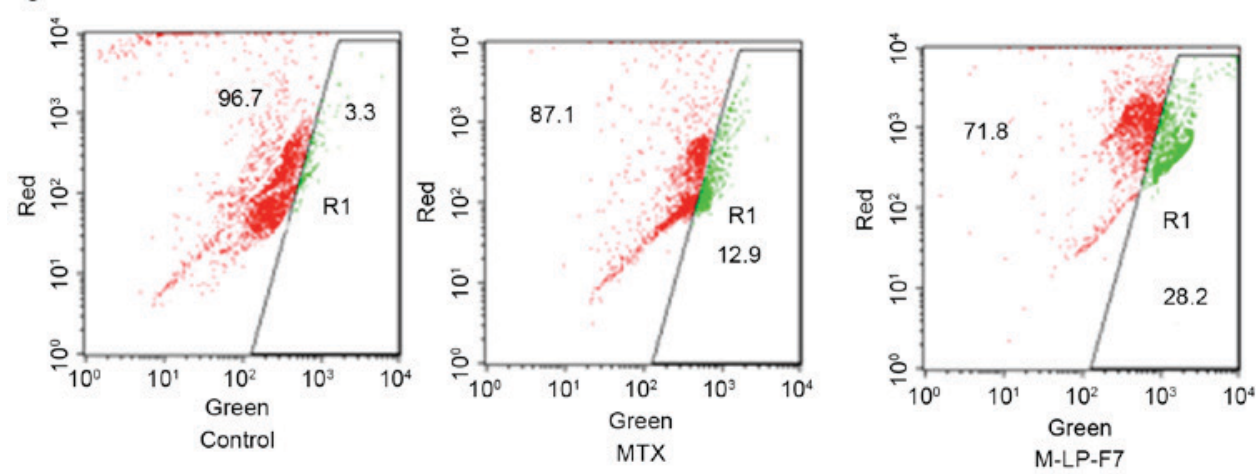

Figure 4. (A) Cell apoptosis in HSC-3 cells after $48 \mathrm{~h}$ of treatment with MTX and M-LP-F7 at equivalent concentration of $70 \mu \mathrm{g} / \mathrm{ml}$ of MTX. (B) Pro-oxidant effect of MTX and M-LP-F7 in HSC-3 prostate cancer cells measured by intracellular ROS using a 2',7'-dichlorofluorescin diacetate assay. (C) Intrinsic apoptotic pathway visible through the change from red to green fluorescence, marking the mitochondrial membrane potential change following treatment with MTX, M-LP-F7 or the control. FITC, fluorescein isothiocyanate; PI, propidium iodine; MTX, methotrexate; M-LP, MTX-entrapped liposomes.

PEG 400 was used as a plasticizer and stabilizer. The cellulose-derived polymers possessed high bio-adhesion properties, which may decrease formulation leakage and disorderliness. The developed films were suitable in terms of mechanical properties and malleability, with good aesthetic and formulation performance (12). Additionally, film bio-adhesion depends on the swelling behavior of the polymers. As the mucoadhesive polymers become hydrated, a proper macromolecular mesh-like biodegradable polymer network formed, which enhanced the interpenetration of polymers and bio-adhesive strength $(35,36)$. In the developed film formulations, HPMC films were the most fragile and easily erodible films due to the highest swelling of the HPMC polymer (37), whereas $\mathrm{CH}$ strengthened the polymer network and reduced the erosion capacity of the films, alongside a controlled release of the drug (38). Films with different polymer concentrations were prepared (F1 to F7) and were characterized based on physiochemical properties. The F7 film was soft, flexible, translucent and had weight uniformity. Thus, F7 film was used to prepare MTX- and M-LP-laden buccal mucoadhesive films.

The cytotoxicity study on HSC-3 cells revealed a marked decrease in the $\mathrm{IC}_{50}$ of MTX in lipid vesicles (M-LP), and further in buccal patch M-LP-F7. This may be attributed to the enhanced permeability of the MTX from M-LP and M-LP-F7 into the cells. The HSC-3 cell line is highly invasive; therefore, the present study aimed to detect the mechanism of MTX-induced apoptosis in HSC3 cells. MTX is active in oral cancer cells, but exhibits marked limitations regarding cell 
uptake and site specificity as it is associated with certain highly toxic effects (17). This was also evaluated via an apoptosis assay in HSC 3 cells; M-LP-F7 increased the percentage of apoptotic cells 3-fold, compared with MTX alone. The results of the assay also suggested that MTX contributes to the intrinsic apoptotic pathway through changing $\Delta \Psi \mathrm{m}$, as evident in the $\mathrm{JC} 1$ assay, an event that is significantly responsible for cancer cell apoptosis. M-LP-F7 was responsible for a 2-fold increase in mitochondrial depolarization. The $\Delta \Psi \mathrm{m}$ disruption was associated with the pro-oxidant effect, which may correspond to ROS accumulation (39). Therefore, intracellular ROS levels were also evaluated in order to detect the expected pro-oxidant mechanism of apoptosis by M-LP-F7. The DCFDA assay indicated an $\sim 68 \%$ peak shift (M2) due to the increased ROS levels in HSC 3 cells caused by M-LP-F7 (containing $70 \mu \mathrm{g} / \mathrm{ml} \mathrm{MTX),}$ in comparison with the positive control $\mathrm{H}_{2} \mathrm{O}_{2}$.

In conclusion, oral mucoadhesive patches for the delivery of MTX liposomes were produced and evaluated in HSC-3 cells to develop a chemotherapeutic delivery system for oral cancer. CH-HPMC-PVA-based mucoadhesive buccal patches exhibited suitable bio-adhesive properties, and prolonged the release of MTX. Oral mucoadhesive patches for oral cancer may be exploited as an effective approach to bypass the limitations of site-specific delivery in oral cancer chemotherapy. Therefore, drug toxicity may be reduced by lowering the dose required, suppressing toxicity and adverse effects.

\section{Acknowledgements}

The present study was supported by the Zhejiang Provincial Education Department (grant no. Y201432814).

\section{References}

1. Gavin A, Pham JT, Wang D, Brownlow B and Elbayoumi TA: Layered nanoemulsions as mucoadhesive buccal systems for controlled delivery of oral cancer therapeutics. Int J Nanomedicine 10: 1569-1584, 2015.

2. Calixto G, Bernegossi J, Fonseca-Santos B and Chorilli M: Nanotechnology-based drug delivery systems for treatment of oral cancer: A review. Int J Nanomedicine 9: 3719-3735, 2014.

3. Strebhardt K and Ullrich A: Paul Ehrlich's magic bullet concept: 100 years of progress. Nat Rev Cancer 8: 473-480, 2008.

4. Deshpande PP, Biswas S and Torchilin VP: Current trends in the use of liposomes for tumor targeting. Nanomedicine (London) 8: 1509-1528, 2013.

5. Chinna Reddy P, Chaitanya KS and Madhusudan Rao Y: A review on bioadhesive buccal drug delivery systems: Current status of formulation and evaluation methods. DARU 19: 385-403, 2011.

6. Smart JD: Buccal drug delivery. Expert opin drug deliv 2: 507-517, 2005.

7. Sudhakar Y, Kuotsu K and Bandyopadhyay AK: Buccal bioadhesive drug delivery-a promising option for orally less efficient drugs. J Control Release 114: 15-40, 2006.

8. Mizrahi B and Domb AJ: Mucoadhesive polymers for delivery of drugs to the oral cavity. Recent Pat Drug Deliv Formul 2: 108-119, 2008

9. Shojaei AH: Buccal mucosa as a route for systemic drug delivery: a review. J Pharm Pharm Sci 1: 15-30, 1998.

10. Adamczak MI, Hagesaether E, Smistad G and Hiorth M: An in vitro study of mucoadhesion and biocompatibility of polymer coated liposomes on HT29-MTX mucus-producing cells. Int J Pharm 498: 225-233, 2016.

11. Nguyen TX, Huang L, Gauthier M, Yang G and Wang Q: Recent advances in liposome surface modification for oral drug delivery. Nanomedicine 11: 1169-1185, 2016.

12. Boddupalli BM, Mohammed ZN, Nath RA and Banji D: Mucoadhesive drug delivery system: An overview. J Adv Pharm Technol Res 1: 381-387, 2010.
13. Shaikh R, Raj Singh TR, Garland MJ, Woolfson AD and Donnelly RF: Mucoadhesive drug delivery systems. J Pharm Bioallied Sci 3: 89-100, 2011.

14. Abruzzo A, Cerchiara T, Bigucci F, Gallucci MC and Luppi B: Mucoadhesive buccal tablets based on chitosan/gelatin microparticles for delivery of propranolol hydrochloride. J Pharm Sci 104: 4365-4372, 2015.

15. Steinberg D and Friedman M: Sustained-release drug delivery of antimicrobials in controlling of supragingival oral biofilms. Expert Opin Drug Deliv 14: 571-581, 2017.

16. Mishra SK, Garud N and Singh R: Development and evaluation of mucoadhesive buccal patches of flurbiprofen. Acta Pol Pharm 68: 955-964, 2011.

17. Abbasi MM, Monfaredan A, Hamishehkar $\mathrm{H}$ and Jahanban-Esfahlan R: New formulated 'DOX-MTX-loaded nanoparticles' down-regulate HER2 gene expression and improve the clinical outcome in OSCCs model in rat: The effect of IV and oral modalities. Asian Pac J Cancer Prev 15: 9355-9360, 2014

18. Abbasi MM, Jahanban-Esfahlan R, Monfaredan A, Seidi K, Hamishehkar $\mathrm{H}$ and Khiavi MM: Oral and IV dosages of doxorubicin-methotrexate loaded-nanoparticles inhibit progression of oral cancer by down-regulation of matrix Methaloproteinase 2 expression in vivo. Asian Pac J Cancer Prev 15: 10705-10711, 2014.

19. Cazzaniga ME, Dionisio MR and Riva F: Metronomic chemotherapy for advanced breast cancer patients. Cancer Lett 400: 252-258, 2017.

20. Costa Lima SA, Gaspar A, Reis S and Durães L: Multifunctional nanospheres for co-delivery of methotrexate and mild hyperthermia to colon cancer cells. Mater Sci Eng C Mater Biol Appl 75: 1420-1426, 2017.

21. Merlano M, Benasso M, Cavallari M, Blengio F and Rosso M: Chemotherapy in head and neck cancer. Eur J Cancer B Oral Oncol 30: 283-289, 1994.

22. Dhanikula RS and Hildgen P: Influence of molecular architecture of polyether-co-polyester dendrimers on the encapsulation and release of methotrexate. Biomaterials 28: 3140-3152, 2007.

23. Alexa IF, Pastravanu CG, Ignat M and Popovici E: A comparative study on long-term MTX controlled release from intercalated nanocomposites for nanomedicine applications. Colloids Surf B Biointerfaces 106: 135-139, 2013

24. Verma DD, Verma S, Blume G and Fahr A: Particle size of liposomes influences dermal delivery of substances into skin. Int $\mathrm{J}$ Pharm 258: 141-151, 2003.

25. Srisuk P, Thongnopnua P, Raktanonchai U and Kanokpanont S: Physico-chemical characteristics of methotrexate-entrapped oleic acid-containing deformable liposomes for in vitro transepidermal delivery targeting psoriasis treatment. Int J Pharm 427: 426-434, 2012.

26. Sharma M, Malik R, Verma A, Dwivedi P, Banoth GS, Pandey N, Sarkar J, Mishra PR and Dwivedi AK: Folic acid conjugated guar gum nanoparticles for targeting methotrexate to colon cancer. J Biomed Nanotechnol 9: 96-106, 2013.

27. Desai KG, Mallery SR, Holpuch AS and Schwendeman SP: Development and in vitro-in vivo evaluation of fenretinide-loaded oral mucoadhesive patches for site-specific chemoprevention of oral cancer. Pharm Res 28: 2599-2609, 2011.

28. Belec L, Tevi-Benissan C, Bianchi A, Cotigny S, Beumont-Mauviel M, Si-Mohamed A and Malkin JE: In vitro inactivation of Chlamydia trachomatis and of a panel of DNA (HSV-2, CMV, adenovirus, BK virus) and RNA (RSV, enterovirus) viruses by the spermicide benzalkonium chloride. J Antimicrob Chemother 46: 685-693, 2000.

29. Kumar R, Verma V, Sharma V, Jain A, Singh V, Sarswat A, Maikhuri JP, Sharma VL and Gupta G: A precisely substituted benzopyran targets androgen refractory prostate cancer cells through selective modulation of estrogen receptors. Toxicol Appl Pharmacol 283: 187-197, 2015.

30. Singh V, Sharma V, Verma V, Pandey D, Yadav SK, Maikhuri JP and Gupta G: Apigenin manipulates the ubiquitin-proteasome system to rescue estrogen receptor- $\beta$ from degradation and induce apoptosis in prostate cancer cells. Eur J Nutr 54: 1255-1267, 2015.

31. Lin CC, Yang JS, Chen JT, Fan S, Yu FS, Yang JL, Lu CC, Kao MC, Huang AC, Lu HF and Chung JG: Berberine induces apoptosis in human HSC-3 oral cancer cells via simultaneous activation of the death receptor-mediated and mitochondrial pathway. Anticancer: res 27: 3371-3378, 2007. 
32. Adrian G and Huang L: Entrapment of proteins in phosphatidylcholine vesicles. Biochemistry 18: 5610-5614, 1979.

33. Sun B and Chiu DT, Determination of the encapsulation efficiency of individual vesicles using single-vesicle photolysis and confocal single-molecule detection. Analytical chemistry 77: 2770-2776, 2005.

34. Akbarzadeh A, Rezaei-Sadabady R, Davaran S, Joo SW, Zarghami N, Hanifehpour Y, Samiei M, Kouhi M and Nejati-Koshki K: Liposome: Classification, preparation, and applications. Nanoscale Res Lett 8: 102, 2013.

35. Peppas NA, Moynihan HJ and Lucht LM : The structure of highly crosslinked poly (2-hydroxyethyl methacrylate) hydrogels. J Biomed Mater Res 19: 397-411, 1985.

36. Peppas NA, Thomas JB and McGinty J Molecular aspects of mucoadhesive carrier development for drug delivery and improved absorption. J Biomater Sci Polym Ed. 20: 1-20, 2009.
37. Perioli L, Ambrogi V, Angelici F, Ricci M, Giovagnoli S, Capuccella $M$ and Rossi C: Development of mucoadhesive patches for buccal administration of ibuprofen. J Control Release 99: 73-82, 2004.

38. Tonglairoum P, Ngawhirunpat T, Rojanarata T1, Panomsuk S, Kaomongkolgit $\mathrm{R}$ and Opanasopit P: Fabrication of mucoadhesive chitosan coated polyvinylpyrrolidone/cyclodextrin/clotrimazole sandwich patches for oral candidiasis. Carbohydr Polym 132: 173-179, 2015.

39. Suski JM, Lebiedzinska M, Bonora M, Pinton P, Duszynski J and Wieckowski MR: Relation between mitochondrial membrane potential and ROS formation. Methods Mol Biol 810: 183-205, 2012. 